\title{
A vibration sensor approach to detect intra- articular needle tip placement in the knee joint: a proof-of-concept study
}

\author{
Rit Apinyankul ${ }^{1}$, Kritsada Siriwattanasit ${ }^{1}$, Kakanand Srungboonmee ${ }^{2^{*}}$, Witchaporn Witayakom ${ }^{1}$ and \\ Weerachai Kosuwon ${ }^{1}$
}

\begin{abstract}
Background: Intra-articular injection in the dry knee joint is technically challenging particularly for the beginners. The aim of this study was to investigate the possible use of the vibration sensor to detect if the needle tip was at the knee intra-articular position by characterizing the frequency component of the vibration signal during empty syringe air injection.

Methods: Two milliliters of air were injected supero-laterally at extra- and intra-articular positions of a cadaveric knee joint, using needles of size 18, 21 and 24 gauge $(G)$. Ultrasonography was used to confirm the positions of needle tip. A piezoelectric accelerometer was mounted medially on the knee joint to collect the vibration signals which were analyzed to characterize the frequency components of the signals during injections.

Results: The vibration frequency band power in the range of $500-1500 \mathrm{~Hz}$ was visually observed to potentially localize the needle tip placement during air injection whether they were at the knee extra-articular or intra-articular positions, as demonstrated by the higher band power (over $-40 \mathrm{~dB}$ or dB) for all the needle sizes. The differences of frequency band power between extra- and intra-articular positions were $18.1 \mathrm{~dB}, 26.4 \mathrm{~dB}$ and $39.2 \mathrm{~dB}$ for the needle size 18G, 21 G and 24G respectively. The largest difference in spectral power was found in the smallest needle diameter (24G).

Conclusions: A vibration sensor approach was preliminarily proved to distinguish the intra-articular from extraarticular needle placement in the knee joint. This study demonstrated a possible implementation of an alternative electronic device based on this technique to detect the intra-articular knee injection.
\end{abstract}

Keywords: Intra-articular knee injection, Needle tip placement, Vibration sensor, Vibration frequency band power

\section{Background}

Intra-articular knee injection is a common procedure in treatment and diagnosis of the knee joint pathology. For example, local anesthesia injection to the knee joint is done in the elective knee arthroscopy cases $[1,2]$ and in some knee physical examinations that need to be done

\footnotetext{
*Correspondence: kakanand.sru@mahidol.edu

${ }^{2}$ Center of Data Mining and Biomedical Informatics, Faculty of Medical Technology, Mahidol University, Nakhonpathom 73170, Thailand Full list of author information is available at the end of the article
}

under anesthesia [3], gadolinium contrast media injection in knee joint imaging [4] and viscosupplement and corticosteroid injection to alleviate the knee pain. These procedures are done as the knee osteoarthritis is one of the most common degenerative joint disease and impacts functional capacity in elderly (prevalence of 3.8\% and 2.7 times more common in women than men) [5]. Disease pathophysiology of knee osteoarthritis involves inflammatory process of synovium and structural changes of articular cartilage including subchondral bone [6]. Most original author(s) and the source, provide a link to the Creative Commons licence, and indicate if changes were made. The images or other third party material in this article are included in the article's Creative Commons licence, unless indicated otherwise in a credit line to the material. If material is not included in the article's Creative Commons licence and your intended use is not permitted by statutory regulation or exceeds the permitted use, you will need to obtain permission directly from the copyright holder. To view a copy of this licence, visit http://creativecommons.org/licenses/by/4.0/. The Creative Commons Public Domain Dedication waiver (http://creativeco mmons.org/publicdomain/zero/1.0/) applies to the data made available in this article, unless otherwise stated in a credit line to the data. 
of the patients suffer from pain, stiffness and impaired joint mobility and often require intra-articular injection with non-steroidal anti-inflammatory drug, corticosteroid or viscosupplement agent for pain relief and inflammation control to improve joint function.

The accuracy of intra-articular needle placement is critical to prevent soft tissue complication and achieve a good treatment outcome by avoiding inadequate analgesia and agent concentration in the intra-articular space. To minimize potential complications of extra-articular injection from local tissue damage, such as atrophy of muscle and subcutaneous fat, pain, skin hypo-pigmentation [7] and skin necrosis [8], proper placement of needle is advocated to confirm intra-articular needle position [9]. Many injection techniques with different knee postures are proposed for agent delivery to the knee, however the best portal and posture is still controversial [10]. A systematic review demonstrates that the superolateral approach in extended knee has the best accuracy around $91 \%$ compared to other approaches in the similar posture (lateral mid-patellar $85 \%$, anteromedial $72 \%$ and anterolateral 67\%) [11]. Additionally, in $90^{\circ}$-bended knee position, a study using squishing technique [12] and post-injection mini air-arthrography [13] demonstrates that injection with modified anterolateral approach has higher accuracy $(89 \%)$ than the superolateral approach (58\%) [14].

No matter what techniques used, the accuracy of needle tip placement is essential, particularly when the knee effusion is not present or the symptomatic dry joint [15]. The extra- or intra-articular position of needle tip can usually be detected by ultrasonography. The ultrasound-guided knee injection and aspiration offers greater accuracy and clinical improvement over using only the conventional landmark technique [16-19], however, the imaging technique requires extra time and the cost of machine prohibits its use in the limited-resource settings. Without an ultrasound machine, the tactile sensation, which is the sensing of tissue resistance felt at the performer's hand, can generally be used to distinguish the needle tip positions in the clinical practice. The lossof-resistance feeling or absence of the backflow when the needle tip in intra-articular, detecting from the tactile feedback felt at the syringe, has also been proposed [20] and implemented as an instrument [21] for the imagingfree technique to increase the intra-articular injection accuracy. However, the learning curve is steep for the technique, especially without the assistance of a device.

Instead of detecting the tissue resistance, this study proposed detecting the vibration of air injection to indicate the needle tip placement. The idea was based on the previous studies that the vibration sensor approach has been used to detect particle flow in the fluidized bed reactor. A fluidized bed reactor, used in many industrial applications, is a device that carries multiphase chemical reactions. Vibration signals, usually taken from the piezoelectric accelerometer mounting outside the fluidized bed reactor, can be used to non-invasively detect the interested particle flow by characterizing the vibration frequency components, for example, to distinguish sandoil-water flow from sand-water flow [22] and to detect the sand flow in the gas-sand flow [23] by observing the power spectral density for particular frequency bands of the vibration signals. Specifically, the sand flow in different media (oil, water [22], gas) exhibits different vibration characteristics. The piezoelectric accelerometer is a surface contact sensor that converts the vibration signal in the form of acceleration of the piezoelectric material inside to the electrical analog signal that can usually be collected by an analog-to-digital system. After transforming those vibration signals into the time-frequency domain commonly by the short-time Fourier transform (STFT) and the wavelet transform [24], different patterns corresponding to the different flow phases, e.g. with or without solid particles in the flow, can be observed and characterized. The vibration detected in the multiphase flow in the fluidized bed reactor comes from the moving interaction between particles in the flow and reflects in the frequency characterization of vibration [24].

When different multiphase flows can be classified using the vibration frequency analysis, detection of injected air flow through different anatomical structures also seems possible. The flow of air injection originated from the needle tip at different anatomical locations might be distinguishable using vibration frequency analysis, suggesting the needle tip placement either intra- or extraarticular positions. The aim of this study was therefore to compare the vibration signals of intra-articular and extraarticular air injection into the cadaveric knee using the piezoelectric accelerometer sensors, in order to characterize the frequency power band of the detected vibration signal. The idea was to generate the vibration due to the flow of air injection and analyze the signal. Specifically, the vibration signals when the injected air flow through the tendon (extra-articular position) should be different from the injected air flow through the knee joint space (intra-articular position) for all the three needle sizes.

\section{Methods}

\section{Experimental setup}

Six consecutive trials of air injection to the knee joint (three needle sizes at two needle tip placements) were done to collect the vibration signals (Fig. 1A). Three needles were the 1.5 -in. $(38-\mathrm{mm})$ thin wall needles of gauge size 18G (HN-1838ET), 21G (HN-2138-ET) and 24G (HN-2438-ET), Nipro needles, 3-9-3, Honjo-Nishi, 


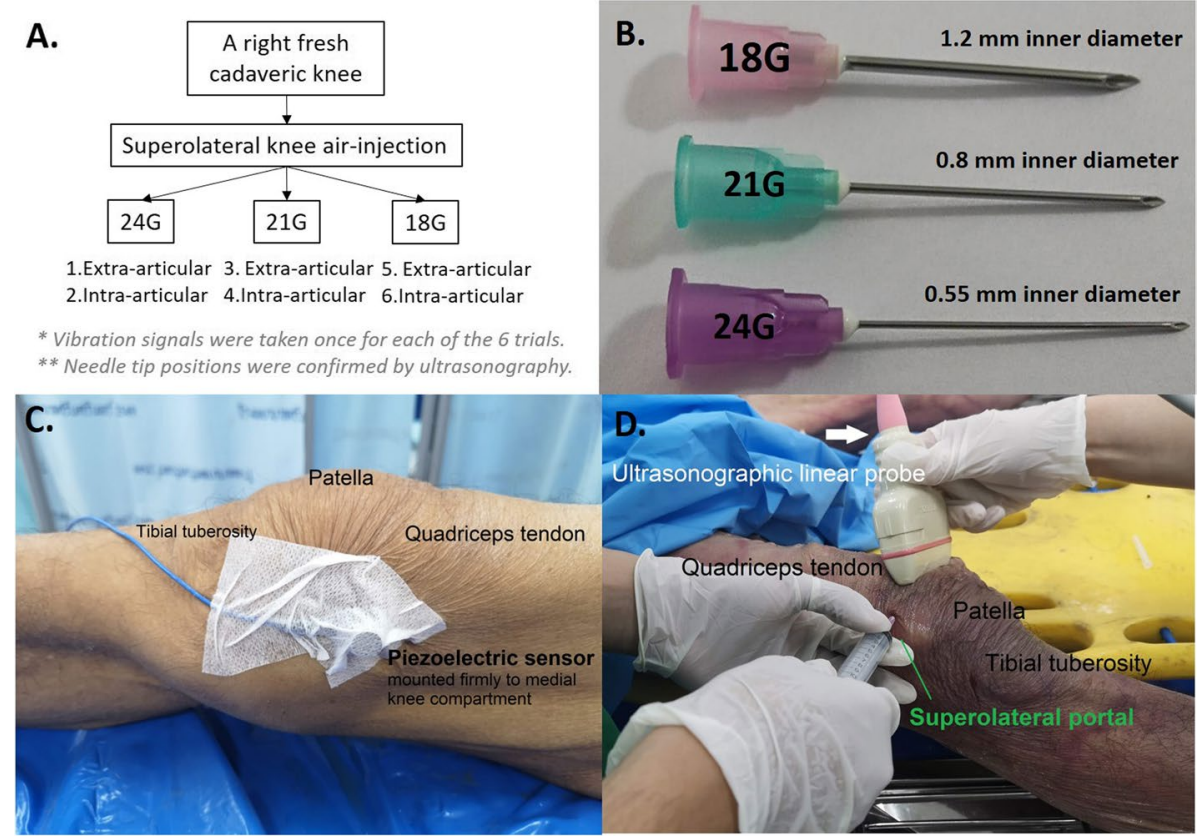

Fig. 1 The workflow of the study consisted of vibration signals measurement for six consecutive trials of the knee injections (A). Three needle sizes were used (B). Experimental setup was done by mounting the accelerometer firmly on the medial collateral ligament just below the medial border of patella, about the mid-patellar level (C). An empty syringe was placed at supero-lateral knee, ready for the 2-ml air injection, and a linear ultrasound probe was positioned on the quadriceps tendon above the patella (D)

Kita-ku, Osaka, Japan (Fig. 1B), all of them were attached to a 10-ml centric tip Nipro luer lock syringe (SY3-xLCEC). The sequence of injection was 24G, 21G and $18 \mathrm{G}$ (smallest to largest diameter to minimize the skin leakage due to needle puncture) with the needle tip at extra-articular following by intra-articular position of each needle. One fresh 68-year-old male cadaver of $168-\mathrm{cm}$ height without any musculoskeletal diseases, preserved at 4 degree Celsius for $48 \mathrm{~h}$ after the congestive heart failure death, was set up for the fully-extended right knee injections at room temperature. A surface-contacting piezoelectric accelerometer sensor (352C33, PCB Piezotronics, USA) was firmly mounted at medial compartment of the knee, approximately at the mid-patellar level on the medial collateral ligament which was just below medial border of patella (Fig. 1C). Ultrasonographic linear probe was used to obtain the ultrasound image in order to confirm whether the needle tip was at extra-articular (quadriceps tendon) or intra-articular (knee joint cavity) position (Fig. 1D). After the desired needle tip position was confirmed by the ultrasound image, two milliliters of the air were injected as fast as possible with superolateral approach from the empty syringe for all cases. The air-flow-induced vibration signals were collected through the sensor (see Acquisition of the vibration signals for details).

\section{Ultrasonographic confirmation of the needle tip positions}

The ultrasonographic linear transducer was used to check for anatomical landmarks of the knee joint. A skillful musculoskeletal radiologist confirmed the positions of needle tip in real-time by using Siemens S3000 ultrasonography machine and linear probe (14 L5), with a frequency between 5.0 and $14.0 \mathrm{MHz}$. The probe was placed on the quadriceps tendon, above the superior pole of the patella (Fig. 1D), and angled so that the quadriceps tendon and articular cartilage of lateral femoral condyle were seen (Fig. 2). The extra-articular position was defined as the needle tip was in the quadriceps tendon (Fig. 2A). The intra-articular position was identified by the space beneath quadriceps tendon and above articular cartilage of lateral femoral condyle (Fig. 2B). Air flow pulse after each injection was also observed from the ultrasound to ensure no needle block occurred during each injection.

\section{Acquisition of the vibration signals}

The vibration signals were collected from the piezoelectric accelerometer (352C33, PCB Piezotronics, USA). The signal was sampled at $10,000 \mathrm{~Hz}(\mathrm{~Hz})$ through an analog-to-digital converter (DEWE-43-A, 24-bit resolution with anti-aliasing filter), connecting to a computer laptop with the data acquisition software (Dewesoft X) to record the data (Fig. 3). The sensitivity 


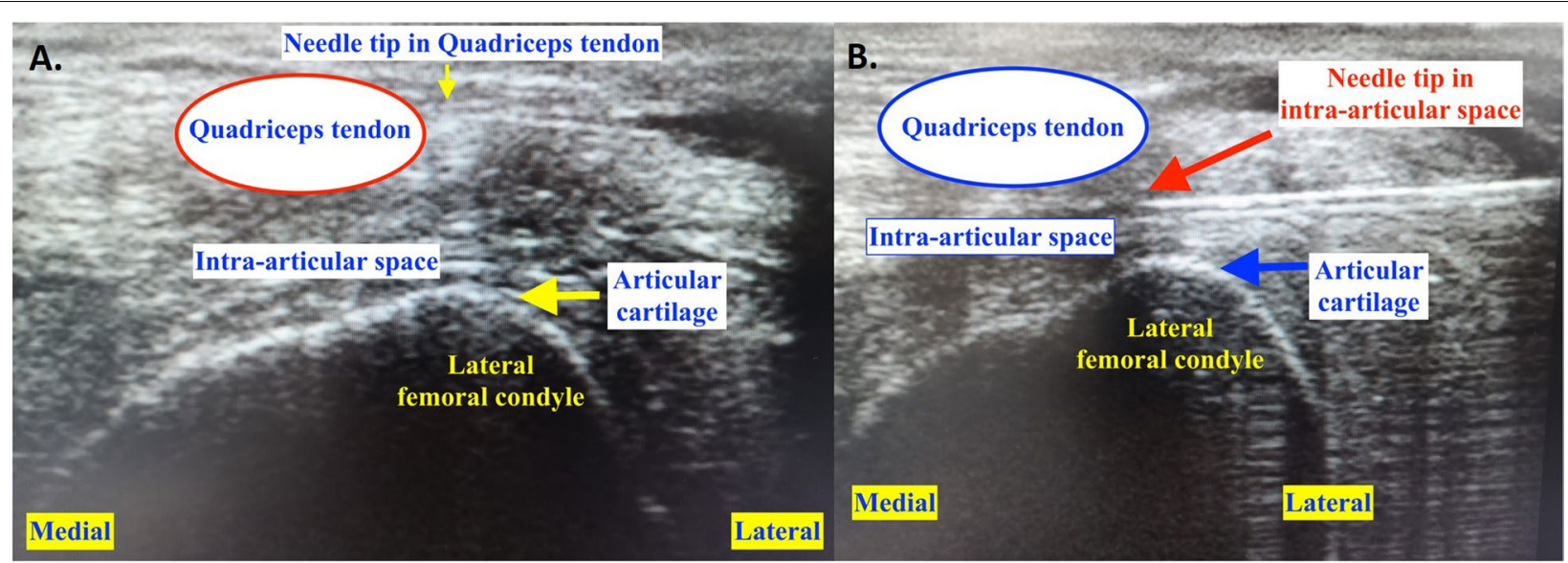

Fig. 2 Ultrasonographic visualization of the needle tip to confirm extra-articular (A) and intra-articular (B) positions. Extra-articular position was defined as the needle tip was at the quadriceps tendon. For intra-articular position, the needle tip was in the intra-articular space beneath the quadriceps tendon and above the articular cartilage of lateral femoral condyle

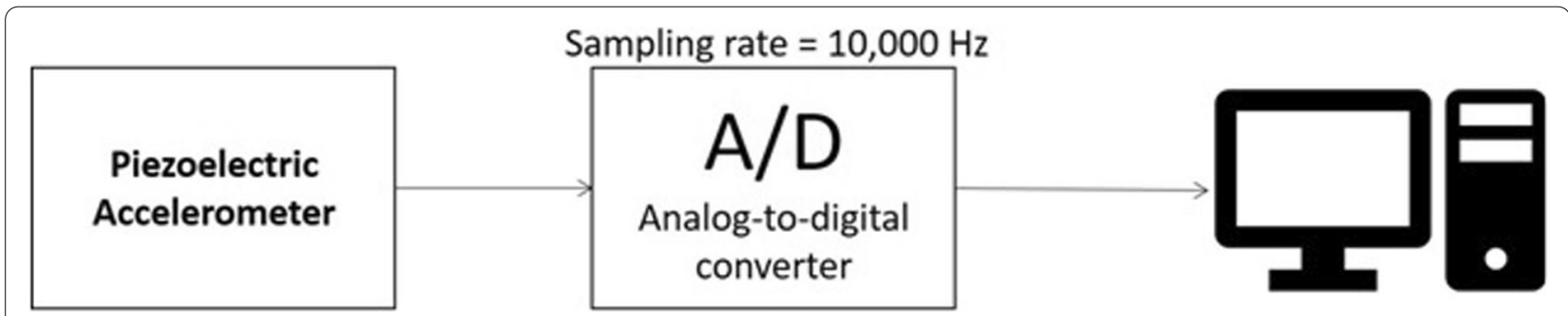

Fig. 3 Schematic diagram of the vibration signal acquisition system. The system consisted of a piezoelectric accelerometer, an analog-to-digital converter with sampling rate of $10,000 \mathrm{~Hz}(\mathrm{~Hz})$ and a computer to collect and display the signals

of accelerometer was $102.9 \mathrm{mV} /$ gravitational acceleration $(\mathrm{mV} / \mathrm{g})$. The vibration signals were recorded in the unit of gravitational acceleration (g) for $3 \mathrm{~s}$. No other filter was applied to the signals.

\section{Signal processing and parameters calculation}

The vibration signals were analyzed in time-frequency domain without additional filter. The short-time Fourier transform was applied to the digital time-domain signal in order to visualize the power spectral density of the signal as time progressing, i.e. the spectrogram. Interested frequency band was determined by visual inspection. Quantification of the frequency band power was done by calculating the summation of the spectral power of the signals in the interested frequency bands after the discrete Fourier transform (256 samples per segmentation with the Hamming window of 250 samples and the overlap of 256 samples) of the signals. All the signal processing was done in MATLAB R2019a (The MathWorks, MA, USA).

\section{Results}

\section{Time frequency response}

The time-frequency responses are displayed in the spectrogram shown in Fig. 4. Higher power spectral density at the high frequency band (approximately $500-1500 \mathrm{~Hz}$ ) was observed when the needle tips were in the knee intra-articular position (bottom row), but not in the extra-articular position (top row). The phenomenon was seen in all the needle sizes (Fig. 4A-C). Specifically, in the high frequency band, minimum vibration power during the extra-articular injection and maximum vibration power during the intra-articular injection were observed from the smallest diameter needle (24G) (Fig. 4C).

\section{Spectral power}

Summation of power spectral density over the interested frequency band (frequency band power) was used to quantify the spectral power. Figure 5 showed the summation of power spectral density in the low $(50-500 \mathrm{~Hz})$ and high $(500-1500 \mathrm{~Hz})$ frequency bands. In the low frequency band, the spectral power was 

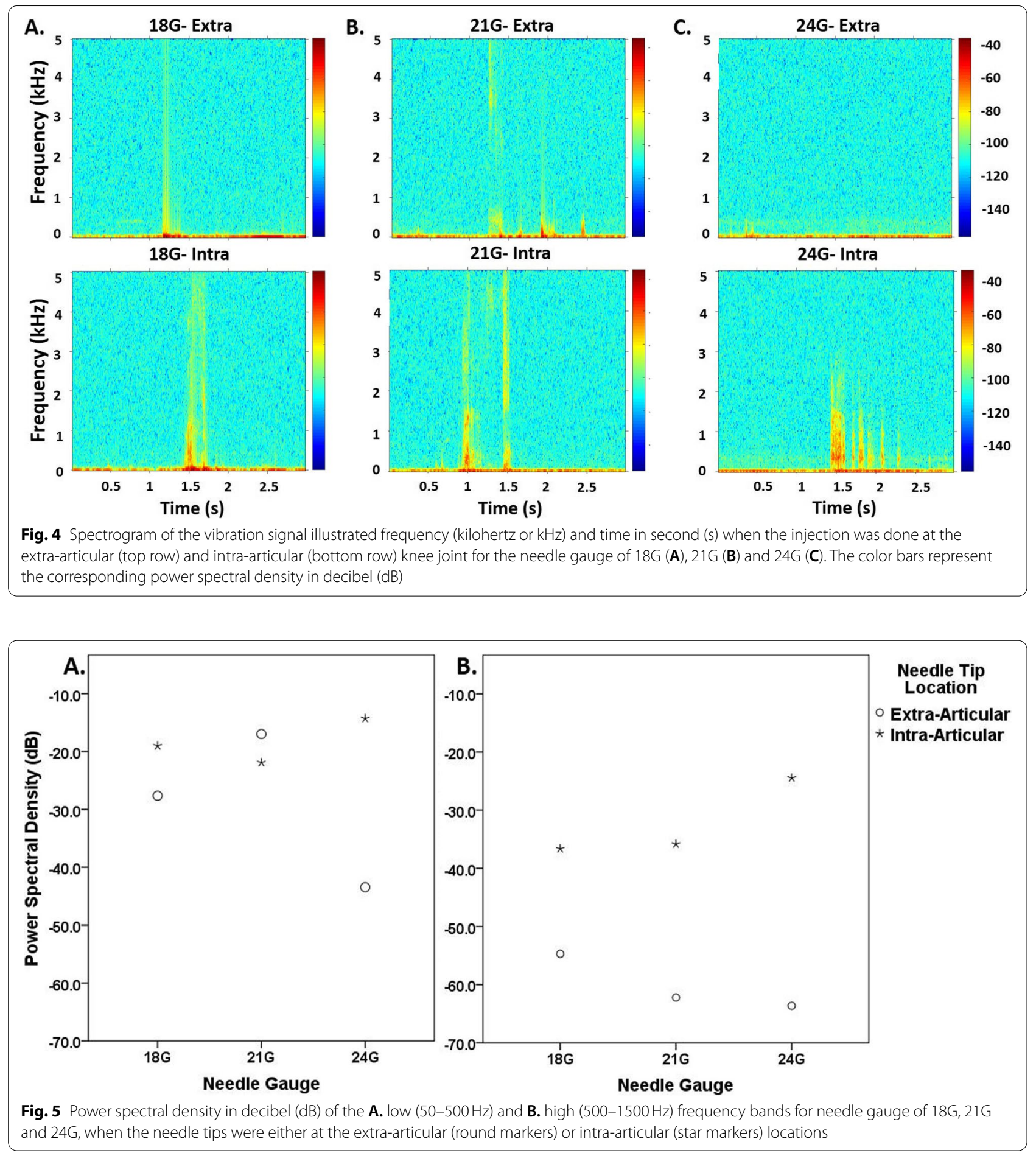

high, approximately over $-40 \mathrm{~dB}(\mathrm{~dB})$ (dark red color of the spectral power in Fig. 4), and it was hard to distinguish between the extra-articular or intra-articular needle tip locations (Fig. 5A). When the needle was intra-articular, higher power spectral density of the high frequency band was observed, compared to the case of the extra-articular needle positions (Fig. 5B). The difference in power spectral density was $18.1 \mathrm{~dB}$, $26.4 \mathrm{~dB}$ and $39.2 \mathrm{~dB}$ for the needle size 18G, $21 \mathrm{G}$ and $24 \mathrm{G}$ respectively. 


\section{Discussion}

Accurate needle tip placement for the knee intra-articular injection is typically challenging for novice physicians especially in a dry knee joint compared to a knee joint with effusion in which the excess synovial fluid makes it easier to confirm the intra-articular position [20]. A fresh cadaver with dry knee joints was therefore used as the study model to prove the concept that the vibration sensor, mounted on the medial side of the knee, can enhance the accuracy of needle tip placement by distinguishing the vibration signal when the needle tip was at the extra- and intra-articular positions of the knee joint during the hard-push air injection using superolateral approach. In clinical practice, the needle tip position can be confirmed by imaging technique like ultrasonography $[18,19]$ or fluoroscopy $[20]$ in which additional machine and the specialist are required to complete the procedure. The image-free approaches include detection of audible squishing sound [12] and feedback tactile sensation [21]. The detection of audible squishing sound during range of motion generated after the small amount of agent injection gives good sensitivity [12] but it could not be practical to move the knee with severe joint pain. The feedback tactile sensation techniques usually rely on the hand sensing such as the loss-of-resistance technique [14] and backflow detection [20], in which the sensing ability depends on the operator's experience and can be challenging for the beginners. Consequently, the special equipment has been proposed to help detect the change in tissue resistance during injection to facilitate the learning of this procedure [21]. This study, therefore, proposed an alternative technique in detecting the vibration of the air injection to locate the needle tip position which can be used either as an equipment to confirm the needle tip placement like the imaging technique or as a feedback tool to practice the hand sensing ability.

The results have shown that the power spectral density in the high frequency band $(500-1500 \mathrm{~Hz})$ in Fig. 5B was more likely to distinguish between the extra- and intraarticular needle tip positions in all needle sizes, compared to the low frequency band $(50-500 \mathrm{~Hz})$ as seen in Fig. 5A. The spectral power in the low-frequency band was generally high for both positions of all the needle sizes (Fig. 5A). Specifically, the intra-articular air flow injection detected from an accelerometer placed at the medial compartment of the knee can therefore be characterized as the higher power spectral density (over $-40 \mathrm{~dB}$ ) in the frequency band of $500-1500 \mathrm{~Hz}$.

Characterizing the vibration signals from the air injection in the knee joint can be similar to the case of vibration monitoring in the fluidized bed from previous studies [22-24]. The distinct frequency components of vibration signals developed from the presence of particle flow is commonly observed in the phase flow in the fluidized bed where the particles velocities can be controlled and the phase flow including bubbles formation can be seen from the side glass window of the chamber. For example, large bubbles and particles-wall interaction usually correspond to low frequency signals [24]. Likewise, in the present study, the power of low frequency components could be due to the air flow that might fluctuate the peri-articular tissues in which it could result from when the injection was at either the extra- or intra-articular sites (Fig. 4). The high frequency signal is usually associated with the air flow [24]. Air flow velocity, bubble formation and bubble size also reflect in the different spectral power of vibration signal. When the air flow is present in the fluidized bed, sharp peak of the power spectrum at $1000 \mathrm{~Hz}$ is found regardless of the flow velocity. But when the air velocity is increasing, higher frequency components gradually appear between 3600 and $4000 \mathrm{~Hz}$. When the frequency components in the range of $800-1500 \mathrm{~Hz}$ appears, it is the sign of bubble formation [24]. The range of $500-1500 \mathrm{~Hz}$ was selected accordingly [24] and based on the observation in Fig. 4 that quantification of this frequency band should be able to distinguish these two needle tip positions. Therefore, high frequency component observed should be explained as the air flow induced vibration in the joint cavity as seen when the needle tip was at intra-articular location during empty syringe air injection, (Figs. 4 and 5).

However, needle sizes may affect the power spectral density of the vibration signal. With the similar volume flow rate in the extra-articular cases, the needle with larger diameter can cause more fluctuation to the periarticular tissues as in the case of the largest diameter needle (18G), where the power spectral density in the high frequency band was higher than other needle sizes (Fig. 5B). For the case of intra-articular 24G needle air injection, smaller cross-sectional flow causing faster flow velocity could also affect higher spectral power (Fig. 5B). Despite the flow velocity, higher spectral power in the high frequency band of the larger diameter needles (18G and $21 \mathrm{G}$ ) could also be due to the moving interaction between the injected air particles and the accumulated air particles from the previous injection. In clinical practice, these three needle sizes are selected for the articular knee injection depending on operators' preferences. The smaller diameter of the needle provides less injection pain to the patients but harder for the clinicians to feel the loss-of-resistance if the technique is solely used to locate the needle tip. An assisted device to enhance the needle tip injection accuracy could be useful for the beginners especially when using the smaller diameter needles. Further study 
with more samples and random sequence of injection is recommended to increase reliability.

Settings that were critical in this study were the location of sensor, the mounting technique and the external vibration. The vibration sensor should be mounted firmly to the flat surface with minimum impact load and external vibration. Relative movement between the sensor and the surface should also be careful as it could produce high spectral power. During injection from the lateral side of the knee, the aforementioned factors should be minimized when placing the sensor on the medial collateral ligament, adjacent to the joint space.

The use of one cadaveric knee sample with single injection per scenario was the major limitation of this study. This was due to the study was done during the COVID-19 pandemic when body donation was restricted. Study in human was possible despite that an advantage of cadaveric study was the absence of needle reflex that could provide the muscle twitch contributing as a small external impact load to the sensor. More cadaveric sample should be obtained after the pandemic. Moreover, there was an effort to minimize the air accumulation in tissue and joint space that could affect the vibration signal in the later trials. That was why the injection was done once in each scenario. Either larger number of cadaveric knees or smaller amount of the air at each injection could help increase number of trials in the future study.

\section{Conclusions}

The superolateral empty syringe air injection technique with a vibration sensor on the medial collateral ligament at the mid-patellar level was proved to distinguish the intraarticular from extra-articular needle placement in the knee joint by considering the spectral power at the frequency band of $500-1500 \mathrm{~Hz}$. This vibration sensor approach could be an alternative technique to detect the accurate location of needle placement. This study demonstrated the possible electronic device implementation of this technique to detect the intra-articular injection when ultrasound machine is not available.

\section{Authors' contributions}

All authors contributed to the study conception and design. Material preparation, data collection and processing were performed by RA, KS and WW. KS performed data analysis and interpretation. The first draft of the manuscript was written by RA and all authors commented on previous versions of the manuscript. All authors read and approved the final manuscript.

\section{Competing interests}

The authors declare that they have no competing interests.

\section{Author details}

'Department of Orthopedics, Faculty of Medicine, Khon Kaen University, Khon Kaen 40002, Thailand. ${ }^{2}$ Center of Data Mining and Biomedical Informatics, Faculty of Medical Technology, Mahidol University, Nakhonpathom 73170, Thailand.
Received: 9 May 2021 Accepted: 2 November 2021

Published online: 15 November 2021

\section{References}

1. Law BK-Y, Yung PS-H, Ho EP-Y, Chang JJH-T, Kwok GK, Fung K-Y, et al. Review of knee arthroscopy performed under local anesthesia. BMC Sports Sci Med Rehabil. 2009;1(1):3. https://doi.org/10.1186/ 1758-2555-1-3.

2. Jacobson E, Forssblad M, Rosenberg J, Westman L, Weidenhielm L. Can local anesthesia be recommended for routine use in elective knee arthroscopy? A comparison between local, spinal, and general anesthesia. Arthroscopy: the journal of arthroscopic \& related. Surgery. 2000;16(2):183-90. https://doi.org/10.1016/S0749-8063(00)90034-3.

3. Malanga GA, Andrus S, Nadler SF, McLean J. Physical examination of the knee: a review of the original test description and scientific validity of common orthopedic tests. Arch Phys Med Rehabil. 2003;84(4):592-603. https://doi.org/10.1053/apmr.2003.50026.

4. Kurra C, Harmon TS, Taylor K, Utz J, Hernandez M, Matteo J, et al. The dark side of gadolinium: a study of Arthrographic contrast at extreme concentrations. Cureus. 2019;11(10):e6006. https://doi.org/10.7759/cureus.6006.

5. Cross M, Smith E, Hoy D, Nolte S, Ackerman I, Fransen M, et al. The global burden of hip and knee osteoarthritis: estimates from the global burden of disease 2010 study. Ann Rheum Dis. 2014;73(7):1323-30. https://doi. org/10.1136/annrheumdis-2013-204763.

6. Jackson DW, Simon TM, Aberman HM. Symptomatic articular cartilage degeneration: the impact in the new millennium. Clin Orthop Relat Res. 2001;391 Suppl:S14-25.

7. Park S-K, Choi YS, Kim H-J. Hypopigmentation and subcutaneous fat, muscle atrophy after local corticosteroid injection. Korean J Anesthesiol. 2013;65(6 Suppl):S59-61. https://doi.org/10.4097/kjae.2013.65.6S.S59.

8. McGee AM, Davison PM. Skin necrosis following injection of non-steroidal anti-inflammatory drug. BJA. 2002;88(1):139-40. https://doi.org/10.1093/ bja/88.1.139.

9. Jones A, Regan M, Ledingham J, Pattrick M, Manhire A, Doherty M. Importance of placement of intra-articular steroid injections. BMJ (Clinical research ed). 1993;307(6915):1329-30. https://doi.org/10.1136/bmj.307. 6915.1329.

10. Maricar N, Parkes MJ, Callaghan MJ, Felson DT, O'Neill TW. Where and how to inject the knee--a systematic review. Semin Arthritis Rheum 2013;43(2):195-203; doi:https://doi.org/10.1016/j.semarthrit.2013.04.010.

11. Hermans J, Bierma-Zeinstra SMA, Bos PK, Verhaar JAN, Reijman M. The most accurate approach for intra-articular needle placement in the knee joint: a systematic review. Semin Arthritis Rheum. 2011;41(2):106-15. https://doi.org/10.1016/j.semarthrit.2011.02.007.

12. Glattes RC, Spindler KP, Blanchard GM, Rohmiller MT, McCarty EC, Block J. A simple, accurate method to confirm placement of intra-articular knee injection. Am J Sports Med. 2004;32(4):1029-31. https://doi.org/10.1177/ 0363546503258703.

13. Bliddal $\mathrm{H}$. Placement of intra-articular injections verified by mini airarthrography. Ann Rheum Dis. 1999;58(10):641-3. https://doi.org/10. 1136/ard.58.10.641.

14. Chernchujit B, Tharakulphan S, Apivatgaroon A, Prasetia R. Accuracy comparisons of intra-articular knee injection between the new modified anterolateral approach and superolateral approach in patients with symptomatic knee osteoarthritis without effusion. Asia-Pacific J Sports Med Arthrosc Rehabil Technol. 2019;17:1-4. https://doi.org/10.1016/j. asmart.2019.02.001.

15. Jackson DW, Evans NA, Thomas BM. Accuracy of needle placement into the intra-articular space of the knee. J Bone Joint Surg Am. 2002;84(9):1522-7. https://doi.org/10.2106/00004623-200209000-00003.

16. Qvistgaard E, Kristoffersen H, Terslev L, Danneskiold-Samsøe B, TorpPedersen S, Bliddal H. Guidance by ultrasound of intra-articular injections in the knee and hip joints. Osteoarthr Cartil. 2001;9(6):512-7. https://doi. org/10.1053/joca.2001.0433.

17. Huang Z, Du S, Qi Y, Chen G, Yan W. Effectiveness of ultrasound guidance on Intraarticular and Periarticular joint injections: systematic review and Meta-analysis of randomized trials. Am J Phys Med Rehabil. 2015;94(10):775-83. https://doi.org/10.1097/PHM.0000000000000260. 
18. Gilliland CA, Salazar LD, Borchers JR. Ultrasound versus anatomic guidance for intra-articular and periarticular injection: a systematic review. Phys Sports Med. 2011;39(3):121-31. https://doi.org/10.3810/psm.2011. 09.1928.

19. WuT, Dong Y, Song HX FY, Li JH. Ultrasound-guided versus landmark in knee arthrocentesis: a systematic review. Semin Arthritis Rheum. 2016;45(5):627-32. https://doi.org/10.1016/j.semarthrit.2015.10.011.

20. Luc M, Pham T, Chagnaud C, Lafforgue P, Legré V. Placement of intraarticular injection verified by the backflow technique. Osteoarthr Cartil. 2006;14(7):714-6. https://doi.org/10.1016/j.joca.2006.02.013.

21. Yao J, Sullivan ST, Eckert CA, Bartlett EC. An orthopedic injection training instrument using flow impedance to indicate needle tip locations. J Clin Monit Comput. 2009;23(6):347. https://doi.org/10.1007/ s10877-009-9202-Z.

22. Wang K, Liu Z, Liu G, Yi L, Liu P, Chen M, et al. Vibration sensor approaches for sand detection in oil-water-sand multiphase flow. Powder Technol. 2015;276:183-92. https://doi.org/10.1016/j.powtec.2015.02.041.
23. Wang K, Liu Z, Liu G, Yi L, Yang K, Li R, et al. Vibration sensor approaches for the sand detection in gas-sand two phases flow. Powder Technol. 2016;288:221-7. https://doi.org/10.1016/j.powtec.2015.11.013.

24. Abbasi M, Sotudeh-Gharebagh R, Mostoufi N, Mahjoob MJ. Non-intrusive monitoring of bubbles in a gas-solid fluidized bed using vibration signature analysis. Powder Technol. 2009;196(3):278-85. https://doi.org/ 10.1016/j.powtec.2009.08.012

\section{Publisher's Note}

Springer Nature remains neutral with regard to jurisdictional claims in published maps and institutional affiliations.
Ready to submit your research? Choose BMC and benefit from:

- fast, convenient online submission

- thorough peer review by experienced researchers in your field

- rapid publication on acceptance

- support for research data, including large and complex data types

- gold Open Access which fosters wider collaboration and increased citations

- maximum visibility for your research: over $100 \mathrm{M}$ website views per year

At BMC, research is always in progress.

Learn more biomedcentral.com/submissions 